\title{
QUANTIFICAÇÃO DA FUNÇÃO ESFINCTERIANA PELA MEDIDA DA CAPACIDADE DE SUSTENTAÇÃO DA PRESSÃO DE CONTRAÇÃO VOLUNTÁRIA DO CANAL ANAL
}

\author{
Luiz Henrique Cury SAAD ${ }^{1}$, Cláudio Saddy Rodrigues $\mathbf{C O Y}^{2}$, \\ João José FAGUNDES², Maria de Lourdes ARIYZONO², \\ Nanako SHOJI $^{3}$ e Juvenal Ricardo Navarro GÓES ${ }^{2}$
}

\begin{abstract}
RESUMO - Racional - Tem sido demonstrado que a pressão máxima de contração voluntária e a pressão média de repouso não refletem a real situação clínica do paciente portador de incontinência fecal, não traduzem a realidade funcional do canal anal, além de poder estar comprometendo a conduta a ser tomada devido ao não-encaminhamento à terapêutica específica. Objetivo - Com a hipótese de que contrair e manter a contração é mais importante que simplesmente contrair, mesmo com pico momentaneamente elevado de pressão, analisou-se a capacidade de sustentação da pressão de contração voluntária do canal anal com o intuito de quantificar a função esfincteriana relativa à continência fecal. Material e Métodos - Submeteram-se a exame manométrico anorretal 72 pacientes (56 mulheres) portadores de incontinência fecal de vários graus e 15 (9 mulheres) indivíduos continentes (normais), avaliando-se a pressão média de repouso, a pressão máxima de contração voluntária e a capacidade de sustentação da pressão de contração voluntária. Resultados - Os indivíduos continentes apresentaram valores normais de pressão média de repouso e de pressão máxima de contração voluntária, além de adequada capacidade de sustentação da pressão de contração voluntária. Os pacientes incontinentes apresentaram pressão média de repouso e pressão máxima de contração voluntária com valores pressóricos normais ou abaixo do normal e perfil semelhante de capacidade de sustentação da pressão de contração voluntária, ou seja, moderada na fase inicial e ruim nas fases intermediária e final, com queda da mesma superior a $35 \%$ em $78 \%$ dos pacientes. A pressão máxima de contração voluntária apresenta excelente especificidade (100\%) porém, sensibilidade baixa (46\%) para incontinência fecal. Comparativamente, a capacidade de sustentação da pressão de contração voluntária apresenta elevadas especificidade (93\%) e sensibilidade (78\%) para incontinência fecal. Embora a pressão máxima de contração voluntária não indique falso-positivos, apresenta $72 \%$ de falso-negativos. A probabilidade deste fato acontecer com a medida de capacidade de sustentação da pressão de contração voluntária é, praticamente, $20 \%$ menor, valor estatisticamente significativo. Conclusão - O indicativo de função esfincteriana é melhor analisado pela capacidade de sustentação. A capacidade de sustentação traduz com mais exatidão, a capacidade funcional do canal anal em relação à continência voluntária, sendo isoladamente, melhor que a pressão máxima de contração voluntária.
\end{abstract}

DESCRITORES - Incontinência fecal. Ânus, fisiologia. Constipação. Manometria. Fadiga muscular.

\section{INTRODUÇ̃̃O}

A incontinência fecal apresenta-se como a perda da habilidade ou da capacidade de limitar a passagem de fezes ou gases em tempo e/ou local socialmente aceitáveis.

Embora a verdadeira incidência seja desconhecida, os estudos têm demonstrado incidência de incontinência fecal de $2 \%$ na população geral, sendo $30 \%$ dos casos acima dos
65 anos de idade e $66 \%$ em mulheres ${ }^{(15)}$. Em indivíduos acima de 70 anos de idade, a incidência de incontinência fecal é de $17 \%{ }^{(21)}$. Estima-se que $3 \%$ das mulheres que tiveram parto vaginal desenvolvem algum grau de incontinência fecal ${ }^{(25)}$.

A continência fecal é adquirida pela combinação de um esfíncter anal competente e fechado, sensibilidade anorretal normal, capacidade e complacência retais adequadas, controle consciente e é mantida tanto quanto a pressão do canal anal seja

\footnotetext{
Departamento de Cirurgia da Faculdade de Medicina de Botucatu da Universidade Estadual Paulista - UNESP. ² Grupo de Coloproctologia da Faculdade de Ciências Médicas da Universidade Estadual de Campinas - UNICAMP. ${ }^{3}$ GASTROCENTRO da Faculdade de Ciências Médicas da Universidade Estadual de Campinas - UNICAMP. Endereço para correspondência: Dr. Luiz Henrique Cury Saad - Praça Isabel Arruda, 157 - $5^{\circ}$ andar - 18602-370 - Botucatu, SP. e-mail: clinsaad@ uol.com.br
} 
superior à pressão retal. Muitos fatores contribuem para a eficácia do canal anal em reter conteúdo colorretal, tais como: o ângulo entre o eixo longitudinal do reto e do ânus, a capacidade e complacência do reto, impedância do enchimento retal promovido por um cólon sigmóide de baixa complacência, diâmetro estreito e alta pressão, a abertura anal semelhante a uma fenda anterior-posterior, a consistência do conteúdo colorretal apresentado ao mecanismo esfincteriano e, principalmente, a contração tônica e voluntária da musculatura esfincteriana. Os músculos do diafragma pélvico ajudam a regular o processo de defecação e a manter a continência, que é mantida parcialmente sob controle voluntário por ação de músculos estriados do esfíncter anal externo e elevador do ânus e, parcialmente, através do sistema nervoso autonômico pelo músculo liso do esfíncter anal interno.

A pressão de contração (PC) é produzida pela contração do esfíncter anal externo e do músculo puborretal, sendo que a PC máxima eleva a pressão do canal anal para mais do que 2 vezes à observada em repouso, podendo ser mantida durante cerca de 50 segundos $^{(8)}$.

A manometria anorretal é um dos testes fisiológicos mais utilizados e estudados, constituindo-se em importante método de investigação utilizado em centros de pesquisa, clínicas ou hospitais especializados, para a avaliação de pacientes com distúrbios anorretais. Tem-se constituído em exame de rotina na investigação de distúrbios relacionados à função de continência e de evacuação, o que tem permitido quantificar vários deles e, ao correlacionar com os dados clínicos, sugerir ou confirmar o diagnóstico e orientar conduta a ser tomada.

Entretanto, usando-se metodologias descritas na literatura, muitos pacientes podem ter distúrbios funcionais relacionados à evacuação ou à continência, e apresentar manometria anorretal normal ${ }^{(18,24)}$. Seria fato a se questionar a validade ou eficácia desse exame em avaliar esses distúrbios ou então, novos dados deveriam ser obtidos e por ele analisados. É possível que os dados obtidos pela manometria anorretal, pelos métodos descritos na literatura, estejam subestimando a incidência de pacientes com incontinência fecal e, conseqüentemente, mais do que fornecer um diagnóstico equivocado, estariam comprometendo a conduta a ser tomada, bem como o resultado final do tratamento ${ }^{(4,14)}$.

Os trabalhos de pesquisa realizados em pacientes incontinentes, praticamente em sua totalidade, têm considerado entre os parâmetros básicos a pressão máxima de contração voluntária (PMCV) e a pressão média de repouso (PMR). A maioria das investigações, portanto, referiam-se a picos momentâneos da $\mathrm{PC}$ voluntária ou à média das pressões de contração ao longo do canal anal ${ }^{(1,3,5,6,9,16,23,26)}$.

Vários estudos na literatura demonstram que pacientes incontinentes apresentavam pressão máxima de repouso e PMCV dentro da faixa de normalidade. Dependendo dos parâmetros utilizados, de $61 \%$ a $100 \%$ dos pacientes incontinentes tinham gradiente de pressão anorretal normal ${ }^{(7,12,20)}$.

Estudos recentes demonstraram preocupação na importância da duração, ou seja, da manutenção da PC quando necessária ${ }^{(13)}$ e que, embora o pico inicial de PC possa ser impressionante, a relevância e a capacidade de aumento momentâneo da PC em adiar ou retardar a evacuação, não estão claras ${ }^{(10)}$.

Diante do exposto, torna-se evidente que a capacidade de retardar ou postergar a evacuação após a presença do estímulo ou sensação de evacuação iminente é função importante dos músculos do diafragma pélvico e fundamental para os mecanismos tanto de evacuação, como de continência. Deste modo, diante de sensação de evacuação iminente, contrair e manter a contração é mais importante do que simplesmente contrair, mesmo que com pico elevado de pressão.

A partir destas constatações e da hipótese de que as medidas de PMR e PMCV esfincteriana não refletem a real situação clínica do paciente, levantou-se a hipótese de ser a capacidade de sustentação (CS) da PC voluntária do canal anal a medida mais correta para a avaliação da continência fecal, no que diz respeito à função de contração esfincteriana.

Este estudo tem o objetivo de analisar a medida da função esfincteriana do canal anal pela determinação da CS da PC voluntária com a hipótese de que este parâmetro indicaria com mais fidelidade a função de contração, do que a PMCV.

\section{MATERIAL E MÉTODOS}

Os pacientes estudados foram avaliados no Laboratório de Fisiologia Anorretal do GASTROCENTRO da Faculdade de Ciências Médicas da Universidade Estadual de Campinas - UNICAMP, Campinas, SP. Incluíram-se 72 pacientes com incontinência fecal em graus variados, desde escapes ocasionais de fezes e de gases, até incontinência franca a fezes sólidas, sendo 56 do sexo feminino e faixa etária entre 27 e 78 anos.

Os indivíduos normais, em número de 15 , foram escolhidos aleatoriamente entre os pacientes encaminhados ao Laboratório de Fisiologia Anorretal devido a outras queixas ou situações, como: proctalgia, prurido anal, pré-operatório de reconstrução de trânsito intestinal e pré-operatório de cirurgia de coluna lombossacra. Os indivíduos normais encontravam-se na faixa etária entre 24 e 72 anos, sendo nove do sexo feminino e não apresentavam queixas de incontinência fecal de qualquer grau.

Foram excluídos pacientes com história de incontinência fecal submetidos a tratamento cirúrgico anterior para este distúrbio, aqueles submetidos a treinamento esfincteriano por biofeedback ou os submetidos a radioterapia pélvica. Também foram excluídos indivíduos normais ou pacientes incontinentes que apresentaram quadro clínico duvidoso quanto ao diagnóstico de incontinência fecal e os valores pressóricos dos parâmetros estudados no exame de manometria anorretal destes indivíduos assemelhavam-se aos valores considerados normais descritos em outros estudos da literatura.

Os pacientes incontinentes e os indivíduos normais foram submetidos a exame manométrico com o uso de sistema de perfusão 
Saad LHC, Coy CSR, Fagundes JJ, Ariyzono ML, Shoji N, Góes JRN. Quantificação da função esfincteriana pela medida da capacidade de sustentação da pressão de contração voluntária do canal anal

contínua, utilizando-se sistema pneumohidráulico de baixa complacência de perfusão capilar contínua (Arndorfer, Inc., GreenValley, WI, EUA), em interface com sistema computadorizado através de software comercialmente encontrado (Gastrosoft, Polygram Lower GI, versão 6.4 e Windows, Synetics Medical Inc., Irwing, TX, EUA e Dynapack MPX 816, Dynamed, São Paulo, SP); foi utilizado cateter axial de oito canais. Os exames foram realizados sem preparo intestinal prévio, uma vez que os pacientes eram incontinentes e muitos referiram ter dificuldade em reter o enema.

\section{DELINEAMENTO}

Após o período de avaliação em repouso, os pacientes foram orientados a promover contração voluntária do canal anal, de forma contínua e ininterrupta, sendo realizado o estudo da sequiência de eventos descritos a seguir:

10 segundos contração voluntária $\{$ Fase inicial

5 segundos mantendo a contração

10 segundos contração voluntária $\{$ Fase intermediária

5 segundos mantendo a contração

10 segundos contração voluntária $\{$ Fase final

mantendo-se o total de 40 segundos de contração voluntária.

Foram analisados os seguintes parâmetros:

- Determinação da PMR

- Determinação da PMCV

... maior valor pressórico encontrado nas três fases de estudo.

- Determinação da CS da PC voluntária

... diminuição do valor pressórico no decorrer das três fases de estudo em relação ao pico inicial da PC voluntária.

Os indivíduos continentes e os pacientes incontinentes foram alocados em quatro grupos (Figuras 1, 2, 3 e 4).

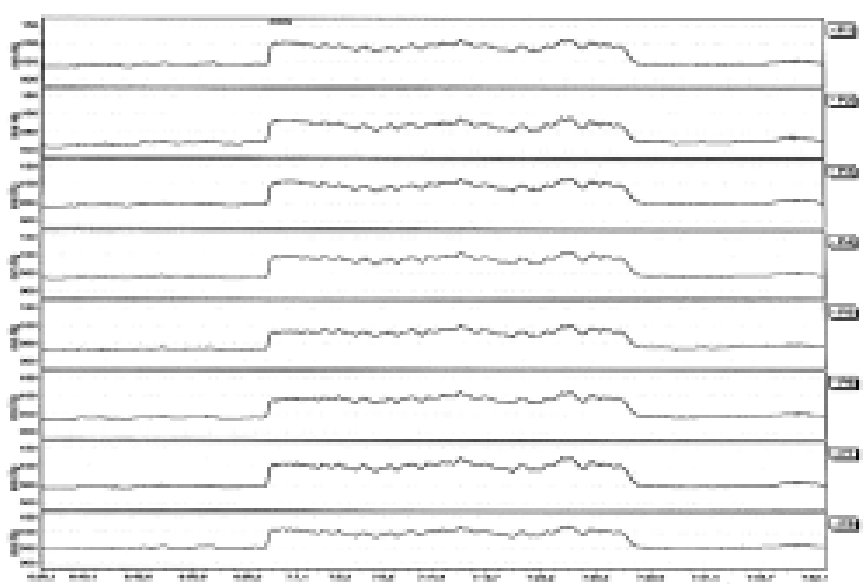

FIGURA 1 - Registro de exame de paciente do grupo I. PMCV normal e adequada CS da contração

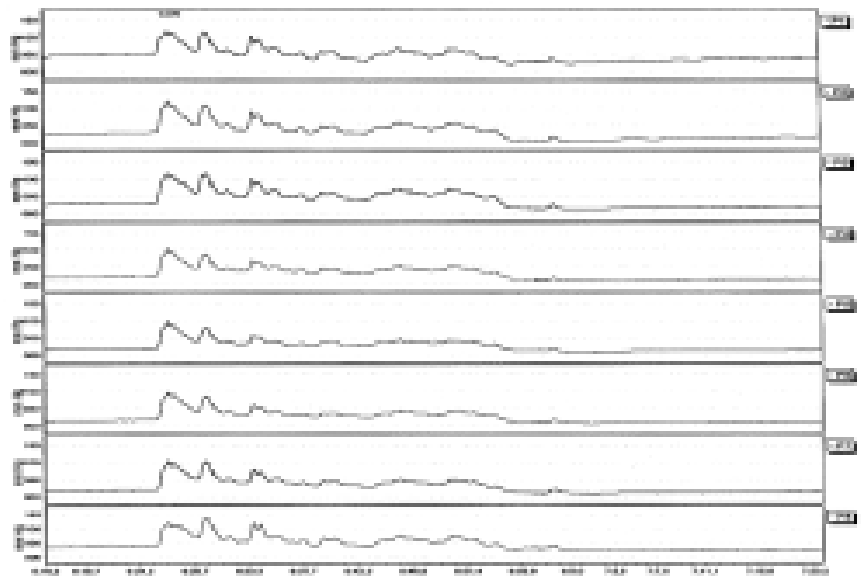

FIGURA 2 - Registro de exame de paciente do grupo II. PMCV normal e inadequada CS da contração

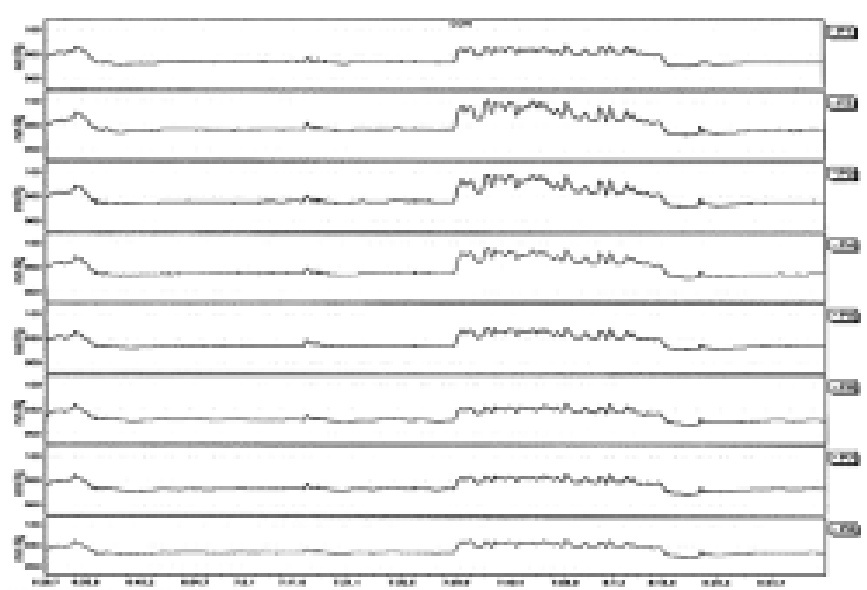

FIGURA 3 - Registro de exame de paciente do grupo III. PMCV abaixo do normal e adequada CS da contração

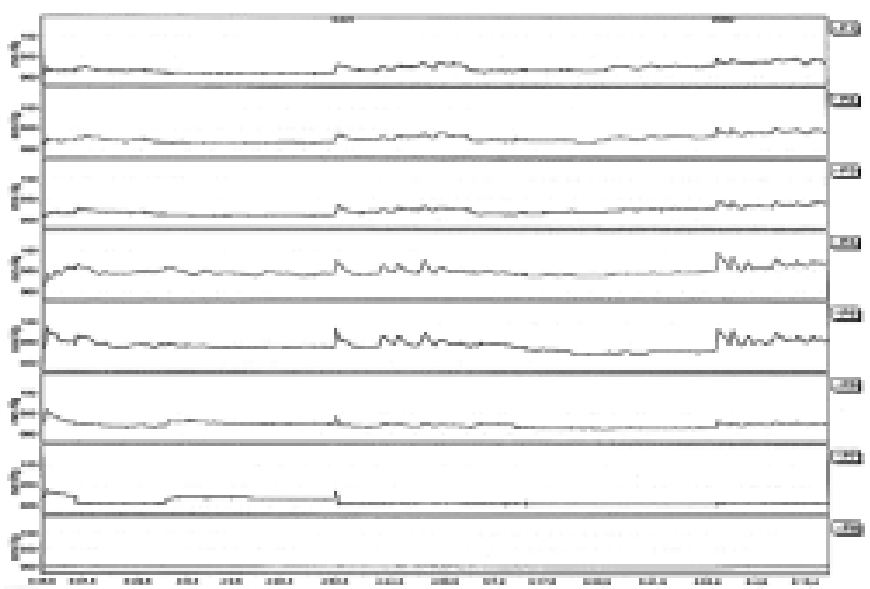

FIGURA 4 - Registro de exame de paciente do grupo IV. PMCV abaixo do normal e inadequada CS da contração 


\section{ANÁLISE ESTATÍSTICA}

Os dados encontrados foram submetidos a análise estatística, com comparação entre proporções de ocorrências pelo teste de Quiquadrado, cálculo de sensibilidade, especificidade, probabilidades de falso-negativo e falso-positivo em tabelas $2 \times 2^{(22)}$.

\section{PROTOCOLO}

Foi seguido protocolo de investigação clínica e para avaliação das queixas de incontinência fecal, avaliou-se o grau clínico, seguindo-se o protocolo descrito abaixo proposto por PINHO et al. ${ }^{(17)}$, com algumas modificações:

\begin{tabular}{cl}
\hline Grau I & $\begin{array}{l}\text { Incontinência para gases } \\
\text { escapes ocasionais (cerca de } 1 / \text { mês), urgência apenas em casos } \\
\text { de diarréia, higiene local sem problemas, sem necessidade de } \\
\text { protetor de roupa, atividades sociais e profissionais preservadas }\end{array}$ \\
\hline Grau II & $\begin{array}{l}\text { Incontinência para gases e fezes líquidas } \\
\text { escapes com maior freqüência (cerca de 1-3/semana), urgência } \\
\text { para fezes consistentes, presença de umidade perianal, } \\
\text { insegurança quanto à higiene anal, uso preventivo de } \\
\text { protetores em alguns casos, comprometimento parcial de } \\
\text { atividades sociais e profissionais }\end{array}$ \\
\hline Grau III & $\begin{array}{l}\text { Escapes diários de fezes. Incontinência para fezes sólidas } \\
\text { urgência fecal, higiene local difícil, necessitando de revisões } \\
\text { freqüentes, uso obrigatório de protetores de roupa atividades } \\
\text { sociais e profissionais impossibilitadas }\end{array}$ \\
&
\end{tabular}

\section{RESULTADOS}

Seguindo o disposto em estudos na literatura, há necessidade de se definir valores normais através de grupos-controle próprios a cada equipamento e laboratório. Os dados obtidos no presente estudo revelaram que no grupo de indivíduos normais, a pressão média de repouso foi de 47 a $84 \mathrm{~mm} \mathrm{Hg}$ (média de 62,70 mm Hg) e a PMCV variou de 130 a $296 \mathrm{~mm} \mathrm{Hg}$ (média de 198,70 mm Hg), havendo nestes indivíduos adequada CS da PC voluntária.

Nos pacientes com incontinência fecal estudados clinicamente, os dados obtidos demonstram que ambas, a PMR e a PMCV, variaram de níveis baixos a normais. Estes dados demonstram que a maioria dos pacientes incontinentes, independente do nível da PMCV apresentada, revelaram perfil semelhante de CS desta pressão: capacidade moderada na fase inicial e ruim nas fases intermediária e final, com queda superior a 35\% do nível inicial de contração na maioria (78\%) dos casos estudados.

A análise dos pacientes incontinentes através da medida da PMCV, mostrou que 39 (54\%) apresentavam níveis normais e 33 (46\%) PMCV abaixo do normal, enquanto que os indivíduos normais apresentaramse em $100 \%$ dos casos com PMCV em nível normal.

Ao se estudarem os pacientes incontinentes, através da CS da contração voluntária, verificou-se que 56 (78\%) deles tinham capacidade inadequada, perfazendo incidência três e meia vezes maior que os pacientes com capacidade adequada (22\%). Os pacientes continentes apresentaram CS adequada em $93 \%$ dos casos.

Ao analisar-se a PMCV nos pacientes incontinentes, verificou-se que os pacientes com grau I, em sua maioria - 12 pacientes $(62 \%)-$ não apresentavam diminuição da medida da PMCV em relação ao valor mínimo normal e nos pacientes que apresentaram diminuição, a queda foi em média de $6 \%$ em relação ao valor mínimo normal da PMCV. Os portadores de grau II, 11 pacientes (30\%), apresentaram diminuição da PMCV, sendo esta queda, em média, de 18\% em relação ao valor mínimo normal. Por fim, os portadores de grau III, quase que em sua totalidade - 14 pacientes $(93 \%)$ - apresentaram diminuição da PMCV, em média 32\% em relação ao valor mínimo da PMCV.

Ao realizar-se a análise da CS da contração voluntária do esfíncter anal externo, no decorrer das três fases de estudo, verificou-se que os pacientes incontinentes apresentaram queda nesta capacidade de $10 \%$ na fase inicial, $31 \%$ na fase intermediária e $40 \%$ na fase final.

A análise da CS, considerando o grau clínico de incontinência fecal, revelou que, nos pacientes com grau I, a queda da CS foi de $21 \%$, nos portadores de grau II, de $30 \%$ e nos de grau III, de $30 \%$. Porém, ao realizar-se a análise comparando o grau clínico e as fases estudadas, constatou-se que na fase final do estudo a CS apresenta queda acima de $35 \%$ nos portadores de incontinência, independentemente do grau clínico. Esta queda foi de $35 \%$ nos portadores de grau I, $45 \%$ nos portadores de grau II e de $43 \%$ naqueles com grau III.

Em relação aos grupos, a queda da CS da PC voluntária, analisada de modo global foi, em média, inferior a $35 \%$ nos quatro grupos: os pacientes do grupo I apresentaram, em média, queda de $13 \%$, os pertencentes ao grupo II, de $32 \%$, os do grupo III, de $14 \%$ e os do grupo IV, de $30 \%$. Ao analisar-se queda da CS em relação aos grupos nas três fases do estudo, verifica-se que esta queda se acentua nas fases intermediária e final. Nos grupos II e IV a queda da CS na fase intermediária é superior a $30 \%$ e superior a $40 \%$ na fase final, verificando-se queda de $47 \%$ no grupo II e $44 \%$ no grupo IV.

Com os dados obtidos, comparando-se a queda da PMCV e a queda CS nos pacientes incontinentes, em relação ao grau clínico de incontinência fecal, verificou-se que os portadores de grau I apresentaram a mesma incidência de queda de PMCV e de CS, ou seja, $40 \%$ dos pacientes apresentaram queda de PMCV e $40 \%$ queda de CS. Nos pacientes com grau III, tanto a PMCV, como a CS apresentaram queda em $93 \%$ dos casos. Nos pacientes portadores de grau II, 92\% apresentaram queda de CS, enquanto a queda de PMCV ocorreu em $30 \%$ destes pacientes .

Ao realizar-se a análise estatística, encontraram-se os resultados apresentados na Tabela 1 .

Em relação à incontinência fecal os efeitos da PMCV e da CS da PC voluntária apresentaram diferenças do ponto de vista estatístico (Tabelas 2 e 3). 
TABELA 1 - Ocorrência de incontinência fecal em relação aos grupos de estudo

\begin{tabular}{lccc}
\hline Grupos & № incontinentes & Proporção & Total \\
\hline Grupo I & 4 & 0,222 & 18 \\
Grupo II & 35 & 0,972 & 36 \\
Grupo III & 12 & 1,000 & 12 \\
Grupo IV & 21 & 1,000 & 21 \\
Total & 72 & 0,827 & 87 \\
\hline$\chi^{2}=58,458$ & $P<0,001$ & Grupo I <(Grupo II = Grupo III = Grupo IV) &
\end{tabular}

TABELA 2 - Efeito da PMCV em relação à incontinência fecal

\begin{tabular}{lccc}
\hline & No continentes & № incontinentes & Total \\
\hline PMCV normal & 15 & 39 & 54 \\
PMCV abaixo normal & 0 & 33 & 33 \\
Total & 15 & 72 & 87 \\
\hline
\end{tabular}

$\chi^{2}=11,076 \quad P<0,001 \quad$ PMCV normal $<$ PMCV abaixo do normal

TABELA 3 - Efeito da CS em relação à incontinência fecal

\begin{tabular}{lccc}
\hline & No continentes & № incontinentes & Total \\
\hline CS adequada $(\mathrm{CS})$ & 14 & 16 & 30 \\
CSN inadequada $\left(\mathrm{CS}^{*}\right)$ & 1 & 56 & 57 \\
Total & 15 & 72 & 87 \\
\hline$\chi^{2}=27,786 \quad P<0,001$ & CS
\end{tabular}

$\chi^{2}=27,786 \quad P<0,001 \quad$ CS adequada $<$ CS inadequada

- Para efeito de predizer a incontinência fecal, considerou-se a medida da PMCV com os parâmetros e resultados abaixo:

$\mathrm{A}=\mathrm{PNI}$ (PMCV abaixo do normal) indicando incontinência

$\mathrm{A}^{*}=\mathrm{PN}(P M C V$ normal $)$ indicando continência

$\mathrm{B}=$ paciente incontinente

$\mathrm{B}^{*}=$ indivíduo continente

$\mathrm{P}=$ resultado

Obtiveram-se os seguintes resultados estatísticos:

\section{Sensibilidade}

Capacidade da medida da PMCV indicar como incontinente indivíduo com incontinência fecal:

$P(A / B)=P(P N I /$ incont.$)=33 / 72=46 \%$

\section{Especificidade}

Capacidade da medida da PMCV indicar como continente indivíduo que é continente:

$$
P\left(A^{*} / B^{*}\right)=P(P N / \text { contin })=15 / 15=1=100 \%
$$

\section{Probabilidade de falso-positivo (FP+)}

Probabilidade de continência mesmo que a medida de PMCV tenha indicado alteração (PNI):

$F P+=P\left(B^{*} / A\right)=P($ contin. $/ P N I)=0 / 33=0=0 \%$

\section{Probabilidade de falso-negativo (FP-)}

Probabilidade de incontinência ainda que a medida da PMCV tenha resultado normal $(\mathrm{PN})$ :

$F P{ }_{-}=P\left(B / A^{*}\right)=P($ incont.$/ P N)=39 / 54=72 \%$

- Para efeito de predizer a incontinência fecal, considerou-se a medida da CS com os parâmetros e resultados abaixo

$\mathrm{A}=\mathrm{CS}^{*}(\mathrm{CS}$ inadequada $)$ indicando incontinência

$\mathrm{A}^{*}=\mathrm{CS}$ (CS adequada) indicando continência

$\mathrm{B}=$ paciente incontinente

$\mathrm{B}^{*}=$ indivíduo continente

$\mathrm{P}$ = resultado

Obtiveram-se os seguintes resultados estatísticos:

\section{Sensibilidade}

Capacidade da medida da CS indicar como incontinente o indivíduo que tem de incontinência fecal:

$P(A / B)=P\left(C S^{*} /\right.$ incont.$)=56 / 72=78 \%$

\section{Especificidade}

Capacidade da medida da CS indicar como continente o indivíduo que é continente:

$$
P\left(A^{*} / B^{*}\right)=P(C S / \text { contin. })=14 / 15=93 \%
$$

\section{Probabilidade de falso-positivo (FP+)}

Probabilidade de continência mesmo que a medida de CS tenha indicado alteração $(\mathrm{CS} *)$ :

$$
F P+=P\left(B^{*} / A\right)=P\left(\text { contin. } / C S^{*}\right)=1 / 57=2 \%
$$

\section{Probabilidade de falso-negativo (FP-):-}

Probabilidade de incontinência ainda que a medida da CS tenha resultado normal (CS):

$F P-=P\left(B / A^{*}\right)=P($ incont.$/ C S)=16 / 30=53 \%$

Em resumo, comparativamente, obteve-se o apresentado na Tabela 4.

TABELA 4 - Comparação entre PMCV e CS

\begin{tabular}{lccc}
\hline Medida & PMCV & CS & \\
\hline Sensibilidade & $46 \%$ & $78 \%$ & $P<0,001$ \\
Especificidade & $100 \%$ & $93 \%$ & nd \\
Falso-positivo & $0 \%$ & $2 \%$ & nd \\
Falso-negativo & $72 \%$ & $53 \%$ & $P<0,001$ \\
\hline
\end{tabular}

\section{DISCUSSÃO}

A importância do esfíncter anal externo para a manutenção da continência e seu papel na fisiopatogenia da incontinência fecal é atualmente aceita e sustentada por várias evidências ${ }^{(11)}$. É evidente que a PMCV baixa e a hipotonia do esfíncter anal externo são acompanhadas de incontinência fecal ou urgência. Chama a atenção, 
entretanto, a freqüência destes achados na maioria dos trabalhos da literatura. RAO e PATEL ${ }^{(19)}$, demonstraram que apenas $53 \%$ dos pacientes incontinentes tinham PMCV abaixo do normal, embora esperasse percentagem maior. RASMUSSEN et al. ${ }^{(20)}$ encontraram que $39 \%$ dos pacientes incontinentes tinham PMCV dentro dos limites normais.

Neste estudo, entre os pacientes continentes, obtiveram-se resultados semelhantes. Entre os pacientes incontinentes, 54\% apresentavam PMCV normal, ou seja, pouco menos da metade destes pacientes (46\%) apresentavam esta pressão abaixo do normal. Esta constatação, na presente série e na literatura, traz consigo o questionamento sobre o valor da PMCV como bom indicativo da função esfincteriana anal.

Sugere-se a CS da PC voluntária como melhor indicativo da função esfincteriana do canal anal, ou seja, a capacidade do indivíduo contrair e manter esta PC voluntária acima de certo nível ou percentagem da pressão inicial, durante determinado período, seria melhor indicativo da função esfincteriana anal do que a PMCV. Portanto, seria mais importante contrair e manter a PC voluntária do que simplesmente contrair, o que refletiria a capacidade do indivíduo em retardar ou postergar a evacuação frente à sensação ou ao estímulo eminente da mesma.

Neste estudo, a CS foi mais fiel quando comparada com a PMCV nos pacientes incontinentes em relação ao grupo controle, mesmo quando comparada em relação ao grau clínico e em relação às fases estudadas.

MARCELLO et al. ${ }^{(10)}$ publicaram estudo referindo-se ao índice de fadiga do esfíncter anal externo. Definiram como taxa de fadiga a variação de contração estacionária pelo período de 40 segundos de contração voluntária e índice de taxa de fadiga a medida calculada do tempo necessário para o esfíncter anal externo tornar-se completamente fatigado. $\mathrm{O}$ esfíncter anal externo está sujeito à fadiga muscular, característica inerente a todos os músculos; mas fadiga implica em que o músculo contraia-se em nível adequado e, após certo tempo, apresente processo de esgotamento, fraqueza, cansaço. Segundo DELISA ${ }^{(2)}$, fadiga é a incapacidade de um músculo de manter ou aumentar a força contra uma resistência, o que implica em esforço repetido, continuado, prolongado, por tempo definido para este músculo. Hoje dispõem-se de aparelhos especiais que podem medir real e fidedignamente, a fadiga ou o tempo de fadiga de um músculo específico. Portanto, tempo de fadiga pode variar de músculo para músculo, bem como para o tipo de contração que este apresente. Como exemplo, o músculo estriado pode apresentar contração lenta ou rápida, isométrica ou não, e o tempo de fadiga difere de acordo com estas características. A melhor avaliação deste comportamento muscular é realizada através da eletromiografia. Não é esta, porém, a situação constatada no presente estudo. Com certeza, a situação fadiga muscular é real e de extrema valia para a pesquisa, avaliação e manuseio da incontinência fecal às custas do esfíncter anal externo.
O que se constata nos pacientes estudados, entretanto, é que ao se solicitar esforço de contração voluntária durante período de 40 segundos, $78 \%$ destes pacientes incontinentes não conseguem sustentar esta contração. Não sustentam mais do que $60 \%$ a $65 \%$ da pressão inicial.

O fato que chama a atenção é que esta característica dos pacientes incontinentes, ou seja, incapacidade de sustentar a contração voluntária, inicia-se no que se define como primeira fase do estudo, os primeiros 10 segundos de contração voluntária e agrava-se nas outras duas fases de estudo. Pela análise global dos pacientes incontinentes houve queda de CS da contração voluntária em média de $10 \%$ na primeira fase (primeiros 10 segundos), $30 \%$ na segunda fase (15 a 25 segundos) e $40 \%$ na terceira fase (30 a 40 segundos).

Este fato torna-se mais evidente ao analisar-se a queda da CS em relação ao grau clínico nas três fases de estudo. Pacientes com grau I apresentaram queda de $35 \%$ na CS na terceira fase de estudo e os portadores de grau II e III, $45 \%$ e $43 \%$, respectivamente. O nível de queda da CS nos pacientes incontinentes é, no mínimo, o triplo na segunda fase de estudo e o quádruplo na terceira fase, independente do grau clínico de incontinência fecal.

Com esta constatação, portanto, do ponto de vista conceitual, não há tempo de fadiga, uma vez que o processo se inicia poucos segundos após o pico de pressão de contração voluntária e agrava-se pelo período de contração de 40 segundos. Portanto, na interpretação do autores, existem duas situações presentes na fisiologia anorretal: a CS da contração voluntária do esfíncter anal externo e a fadiga muscular deste esfíncter. Quando os pacientes incontinentes não conseguem sustentar a contração voluntária desde o início da solicitação, ou seja, desde o início do processo, isto caracteriza incapacidade deste músculo, do esfíncter e, portanto, não há fadiga, mas sim incapacidade.

A taxa de fadiga é novo indicativo da função anorretal que tem sido aplicada e estudada e, para tanto, tem se mostrado de grande valia. Acredita-se, porém, que a alteração na função anorretal de continência envolva processo mais precoce e anterior à fadiga muscular. É possível que os pacientes incontinentes apresentem as duas situações. Qual delas seria a mais freqüente nestes pacientes, ou qual refletiria mais fielmente a incontinência, são questionamentos válidos e que induzem a novas pesquisas e a novos questionamentos.

\section{CONCLUSÃO}

Os dados obtidos neste estudo permitem concluir que, independentemente dos valores da PMCV serem normais ou baixos, pacientes com baixa capacidade de sustentação esfincteriana apresentaram os piores graus de incontinência fecal; independentemente do grau clínico 
Saad LHC, Coy CSR, Fagundes JJ, Ariyzono ML, Shoji N, Góes JRN. Quantificação da função esfincteriana pela medida da capacidade de sustentação da pressão de contração voluntária do canal anal

estudado e dos valores de PMCV, a baixa CS de contração esfincteriana traduziu com mais exatidão a capacidade funcional do canal anal quanto à continência voluntária; a medida da $\mathrm{PMCV}$ apresenta alta especificidade e baixa sensibilidade para incontinência fecal; a medida da CS da contração voluntária apresenta alta especificidade e alta sensibilidade para incontinência fecal; a quantificação da CS da pressão de contração voluntária é bom índice de função esfincteriana anorretal, sendo isoladamente, melhor que a medida de PMCV.

Saad LHC, Coy CSR, Fagundes JJ, Ariyzono ML, Shoji N, Góes JRN. Sphincteric function quantification by measuring the capacity to sustain the squeeze pressure of the anal canal. Arq Gastroenterol 2002;39(4):233-239.

ABSTRACT - Background - It has been demonstrated that the maximum squeeze pressure and the mean resting pressure do not reflect the true clinical situation of patients having fecal incontinence, as well as the functional status of the anal canal. Furthermore, a wrong diagnosis could be obtained and therefore misleading to a not effective treatment. Aim - Under the hypothesis that squeezing and sustaining the anal canal contraction is more important than the maximum squeeze pressure, the capacity to sustain the squeeze pressure of the anal canal was analyzed aiming to quantify the sphincteric function. Methods - Seventy-two patients having fecal incontinence in different degrees (56 female) and 15 normal individuals ( 9 female) were submitted to anorectal manometry to measure the mean resting pressure, the maximum voluntary squeeze pressure and the capacity to sustain the squeeze pressure. Results - Normal individuals had normal values of mean resting pressure and maximum squeeze pressure, and adequate capacity to sustain the squeeze pressure of the canal anal. Incontinent patients had mean resting pressure and maximum squeeze pressure with normal or below normal pressoric values and similar profile of capacity to sustain which was moderate in the initial phase and worse in the intermediate and final phases, with decreasing of the capacity to sustain more than $35 \%$ in $78 \%$ of the patients. The maximum squeeze pressure presented excellent specificity (100\%), but low sensitivity (46\%) for fecal incontinence. Comparatively, the squeeze pressure presented high specificity (93\%) and high sensitivity $(78 \%)$ for fecal incontinence. Although the maximum squeeze pressure did not indicate false positive, it presented a $72 \%$ false negative. The probability of this event to happen with the capacity to sustain measure is $20 \%$ lower, and it was statistically significant. Conclusion - Sphincteric function can be better analyzed by using the capacity to sustain the squeeze pressure. capacity to sustain indicates more precisely the functional capacity of the anal canal in relation to voluntary continence, and it is better than maximum squeeze pressure as an isolated index.

HEADINGS - Fecal incontinence. Anus, physiology. Constipation. Manometry. Muscle fatigue.

\section{REFERÊNCIAS BIBLIOGRÁFICAS}

1. Cali RL, Blatchford GJ, Perry RE, Pitsch RM, Thorson AG, Christensen MA Normal variation in anorectal manometry. Dis Colon Rectum 1992;35:1161-4.

2. Delisa JA. Exercícios terapêuticos. In: Delisa, JA. Tratado de medicina de reabilitação. São Paulo: Manole; 1993. p. 401-30.

3. Enck P, Kulhbusch R, Lubke H, Frieling T, Erckenbrecht JF. Age and sex and anorectal manometry in incontinence. Dis Colon Rectum 1989;32:1026-30.

4. Felt-Bersma RJ, Klinkenberg-Knol EC, Meuwissen SG. Anorectal function investigations in incontinent and continent patients. Differences and discriminatory value. Dis Colon Rectum 1990;33:479-85.

5. Felt-Bersma RJ, Meuwissen SGM. Anal manometry. Int J Colorectal Dis 1990;5:170-3

6. Hill J, Corson RJ, Brandon H, Redford J, Faragher EB, Kiff ES. History and examination in the assessment of patients with idiopathic fecal incontinence. Dis Colon Rectum 1994;37:473-7.

7. Jacobs PP, Scheuer M, Kuljer JH, Vingerhoets MH. Obstetric fecal incontinence. Role of pelvic floor denervation and results of delayed sphincter repair. Dis Colon Rectum 1990;33:494-7.

8. Jorge JMN, Wexner SD. Etiology and management of fecal incontinence. Dis Colon Rectum 1993;36:77-97.

9. Jorge JMN, Wexner SD. A practical guide to basic anorectal physiology investigations. Contemporary Surgery 1993;43:214-24.

10. Marcello P, Barrett RC, Coller JA, Schoetz DJ Jr, Roberts PL, Murray JJ, Rusin L. Fatigue rate index as a new measurement of external sphincter function. Dis Colon Rectum 1998:41:336-43.

11. Mavrantonis CMD, Wexner SD. Clinical approach to fecal incontinence. J Clin Gastroenterol 1998;27:108-21.

12. McHugh SM, Diamant SE. Effects of age, gender and parity on anal canal pressures. Contribution of impaired anal sphincter function to fecal incontinence. Dig Dis Sci 1987;32:726-36.

13. Mitrani C, Chun A, Desautels S, Wald A. Anorectal manometric characteristics in men and women with idiopathic fecal incontinence. J Clin Gastroenterol 1998;26:175-8.
14. Morgado PJ Jr, Werner SD, James K, Nogueras JJ, Jagelman DG. Ileal pouch-anal anastomosis is preoperative anal manometry predictive of postoperative functional outcome? Dis Colon Rectum 1994;37:224-8.

15. Nelson R, Norton N, Cautley E, Furner S. Community-based prevalence of anal incontinence. JAMA 1995;274:559-61.

16. Pedersen IK, Christiansen J. A study of the physiological variation in ana manometry. Br J Surg 1989;76:69-71.

17. Pinho M. Incontinência fecal. In: Cruz GMG, editor. Coloproctologia. Rio de Janeiro: Revinter; 2000. v.2, p. 1250-62.

18. Rao SS. Manometric evaluation of defecation disorders - Part II. Fecal incontinence. Gastroenterologist 1997;5:99-111.

19. Rao SS, Patel RS. How useful are manometric tests of anorectal function in the management of defecation disorders? Am J Gastroenterol 1997;92:469-75.

20. Rasmussen OO, Rinholt C, Alstrup N, Christiansen J. Anorectal pressure gradient and rectal compliance in fecal incontinence. Int $\mathbf{J}$ Colorectal Dis 1998;13:157-9.

21. Resnick NM, Beckett LA, Branch LG, Scherr PA, Wetle T. Short term variability of self report of incontinence in older persons. J Am Geriatr Soc 1994;42:202-7.

22. Rosner B. Fundamental of biostatistics. 2. ed. Boston: Duxbrery; 1986. p. 54-8

23. Sangwan YP, Coller JA, Barrett RC, Roberts PL, Murray JJ, Schoetz DJ Jr. Can manometric parameters predict response to biofeedback therapy in fecal incontinence? Dis Colon Rectum 1995;38:1021-5.

24. Siproudhis L, Bellisant E, Pagenault M, Mendler MH, Allain H, Bretagne JF, Gosselin M. Fecal incontinence with normal anal canal pressure: where is the pitfall? Am J Gastroenterol 1999;94:1556-63.

25. Snooks SJ, Swash M, Stechell M. Injury to innervation of pelvic floor sphincter musculature in childbirth. Lancet 1984;2:546-50.

26. Willianson JL, Nelson RL, Orsay C, Pearl R, Abcarian H. A comparison of simultaneous longitudinal and radial recordings of anal canal pressures. Dis Colon Rectum 1990;33:201-6. 